\title{
High-speed fracture behaviour of glass bottles when using underwater shockwaves
}

\author{
H. Sakamoto ${ }^{1}$, S. Kawabe ${ }^{2}$ \& S. Itoh ${ }^{1}$ \\ ${ }^{1}$ Graduate School of Science and Technology, \\ Kumamoto University, Japan \\ ${ }^{2}$ Kumamoto Prefecture Technical College, Japan
}

\begin{abstract}
The high-speed fracture process of glass bottles when using underwater shockwaves and the glass bottle recycling technology that uses this crushing technique are discussed. The proposed technique is an efficient method for the smashing and washing of glass bottles. The production cost of producing cullet, which is small glass fragments, can be decreased as a result. In this study, using commercial beer bottles, the relationship between the cullet size and various explosive conditions were clarified. Moreover, the behaviour of the underwater shockwaves was observed by the framing photograph method, the shadowgraph method and the illumination photography method, and the FEM simulation was executed in order to clarify the high-speed fracture mechanism.
\end{abstract}

Keywords: glass-cullet, underwater shockwave, high-speed fracture.

\section{Introduction}

Bottles made of glass are used voluminously as containers for water, alcoholic drinks and medicine. Many of these glass bottles are re-used as returnable bottles or recycled as the raw material for other glass bottles, in which case it is called 'cullet', being the small crushed fragment of the glass. Recycling by conventional methods in a large-scale crushing machine is expensive and therefore the ratio of this production method becomes lower in comparison with that of the recycling as returnable bottles. In the case of medicine bottles, almost all of them are dumped as landfill dust [1].

In this study the authors paid attention to the recycling technique for crushing the glass bottles by using an underwater shockwave [2-5]. When this technology 
is used, the recycling cost of glass bottles falls because of the execution of the crushing and washing process simultaneously. This proposed method has a lot of advantages in comparison with a conventional large-scale crushing machine:

1. The crushed fragments of glass do not scatter outside and can be collected completely because the crushing work is done in the water container.

2. Washing is unnecessary before the crushing and the cullet is washed completely. This method can also be applied to medicine glass containers because they are washed at the same time as crushing.

3 . The crushing and washing operation can be done simultaneously.

Therefore, it is necessary to clarify the high-speed fracture behaviours of the glass bottles by underwater shockwaves quantitatively for the commercialisation of this method.

In this report, the relation between the strength of underwater shockwaves and fracture cullet grain size are discussed. Moreover, the behaviour of the underwater shockwaves was observed by the framing photograph method, the shadowgraph method and the illumination photography method, and the FEM simulation was executed in order to clarify the high-speed fracture mechanism.

\section{Experiment}

\subsection{Experimental method}

In the experiment, glass bottles were subjected to underwater shockwaves and crushed into small fragments. The cullet was collected and classified into four kinds of sizes. Each size of cullet was measured and arranged by the weight distribution.

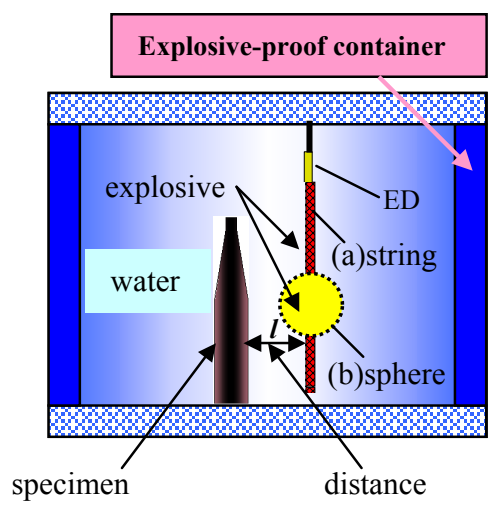

Figure 1:
Scheme of explosion proof container.

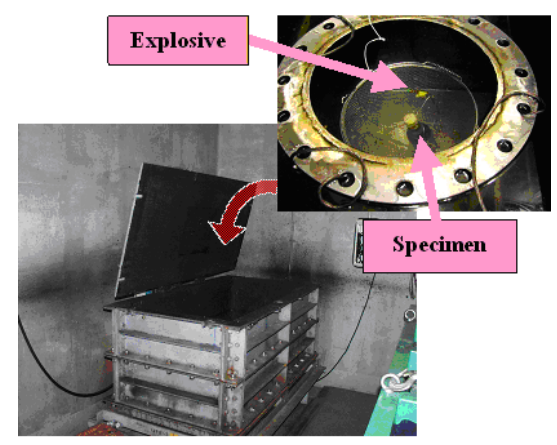

Figure 2: Experimental apparatus.

The specimen and explosive, (a) string type and (b) sphere type, were set in the explosive container shown in Fig.1. The experiment was done in the explosion-proof steel container, which had been filled with water. This container 
is stored in the experimental apparatus because of safety. Figure 2 shows the photograph of the experimental apparatus.

\subsection{Specimen and experimental conditions}

We used commercial beer bottles $(500 \mathrm{ml})$ as the specimen shown in Fig.3. The inside of the bottle was charged with air and was fixed at the middle of the container shown in Fig.1.

The distance $l$ shown in Fig. 1 of the explosive from the bottle has been changed in order to examine the effect on the fragment grain sizes. The five distances from the explosive to the bottle were determined as follows: $l=$ $100 \mathrm{~mm}, 50 \mathrm{~mm}, 40 \mathrm{~mm}, 30 \mathrm{~mm}$ and $20 \mathrm{~mm}$. The PENTHRIT (explosive rate: 6308 $\mathrm{m} / \mathrm{s}$ ) was used as the explosive and the electric detonator was used as the ignition.

The experiment was executed in the conditions shown in Table 1.

\subsection{Optical observation}

In order to visualize the bottle fracture caused by underwater shockwaves, the behaviours of the underwater shockwaves were observed by the framing photograph method, the shadowgraph method and illumination photography method. A high-speed camera with two xenon flashlights was used. The outline of the optical observation system is shown in Fig.4.

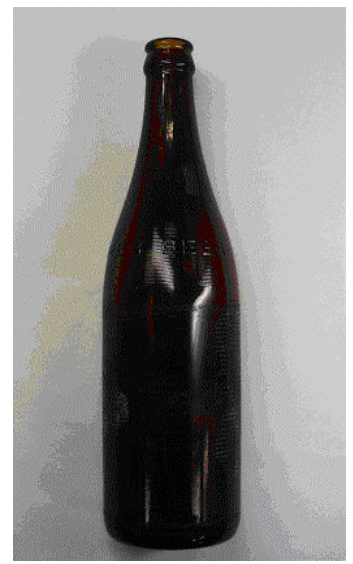

Figure 3: $\quad$ Specimen (beer bottle $(500 \mathrm{ml})$ ).

Table 1: $\quad$ Experimental conditions.

\begin{tabular}{|l|l|l|}
\hline & \multicolumn{1}{|c|}{ A } & \multicolumn{1}{c|}{ B } \\
\hline Shape of explosive & String & Sphere \\
\hline Inside of bottle & Air & Air \\
\hline Kind of explosive & PENTHRIT & PENTHRIT \\
\hline
\end{tabular}




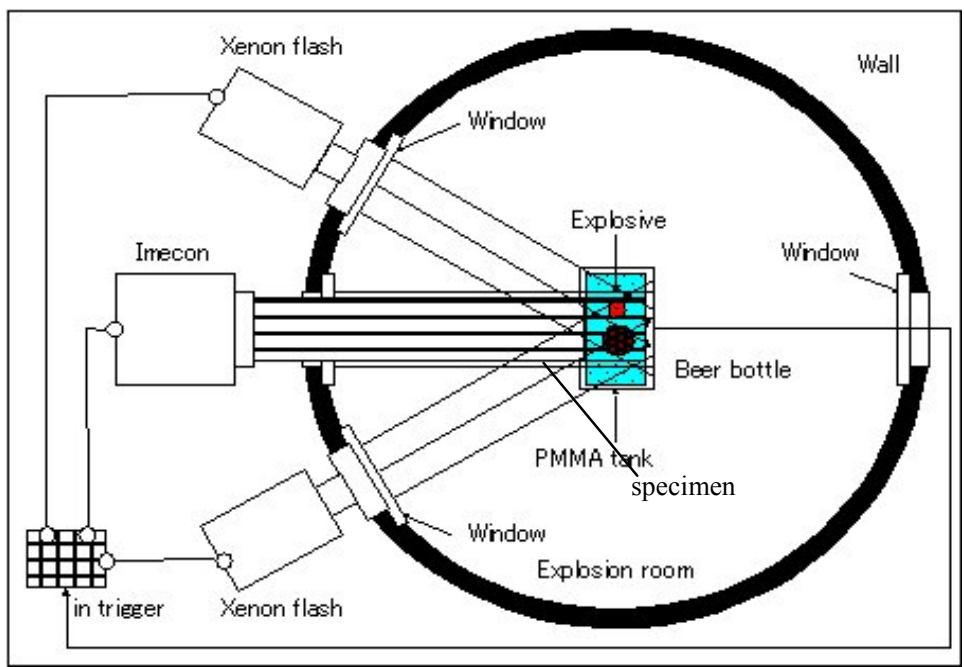

Figure 4: The outline of the optical observation system.

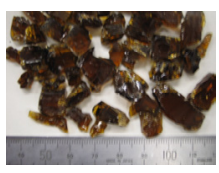

(a) $>4.75 \mathrm{~mm}$

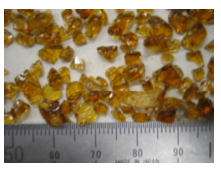

(b) $4.75 \sim 2 \mathrm{~mm}$

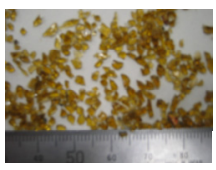

(c) $1 \sim 2 \mathrm{~mm}$

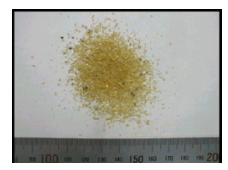

(d) $<1 \mathrm{~mm}$

Figure 5: $\quad$ Size and shape of the cullet.

\section{Results and discussion}

After the explosive experiments, the fragments of the glass bottle were collected and dried. The dried cullet fragments were classified by sieving and each weight was measured. Three kinds of sieves of $4.75 \mathrm{~mm}, 2 \mathrm{~mm}$ and $1 \mathrm{~mm}$ were used in order to classify the cullet sizes such as more than $4.75 \mathrm{~mm}, 2 \mathrm{~mm}-4.75 \mathrm{~mm}, 1$ $\mathrm{mm}-2 \mathrm{~mm}$ and less than $1 \mathrm{~mm}$.

The photographs of the classified cullet are shown in Fig.5. The cullet weight distributions in the case of string type at condition A are shown in Fig. 6. From this figure, in the case of a distance of $20 \mathrm{~mm}$ from the explosive and the bottle, it is found that the weight ratio of the grain size is less than $1 \mathrm{~mm}$ and is the highest of all, and in the case of a distance of $100 \mathrm{~mm}$, that of the grain size of less than $1 \mathrm{~mm}$ is the lowest. It is interesting that the weight distribution of $1-2 \mathrm{~mm}$ grain sizes has not changed much regardless of the distance of the explosive and the bottle. Comparing the effect of the explosive configuration on the cullet size, the weight ratio of the sphere type explosive of less than $1 \mathrm{~mm}$ is more than that of the string type explosive. 
In order to visualize the bottle fracture by shockwave, the framing photograph was taken by using an Image Converter High-Speed Camera (IMECOM 468) and Xenon flash light (H1/20/50 type). Figures 7 and 8 show the shockwave propagation by the Image converter in the case of (a) string type and (b) sphere

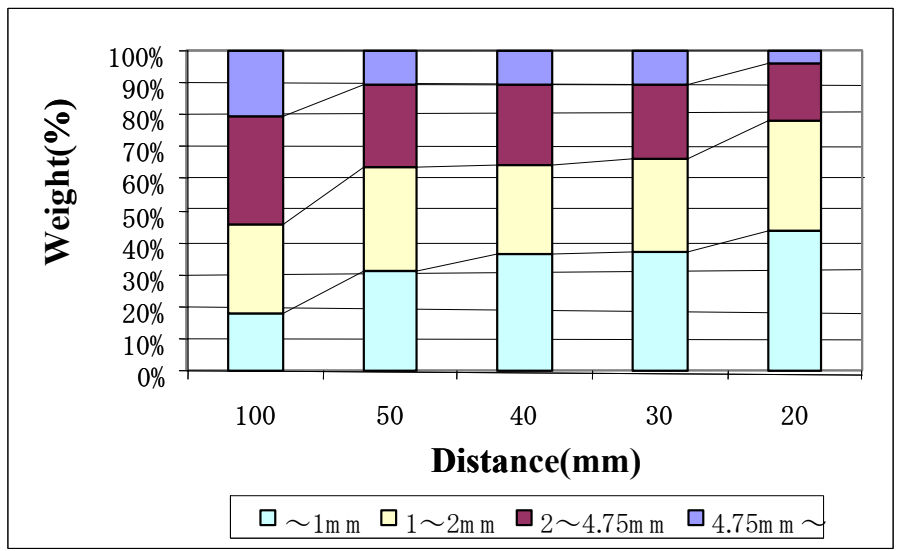

Figure 6: Cullet size distributions in difference of distance from the explosive to the bottle (string type).

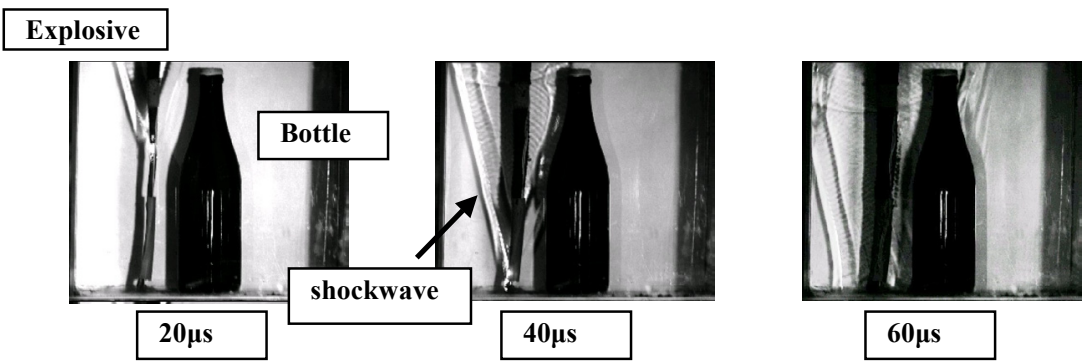

Figure 7: Shockwave propagation (string type).

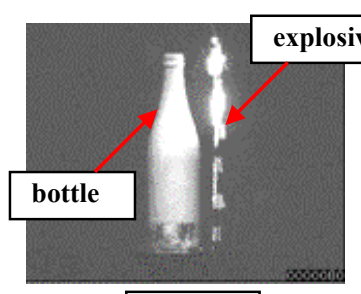

$80 \mu s$

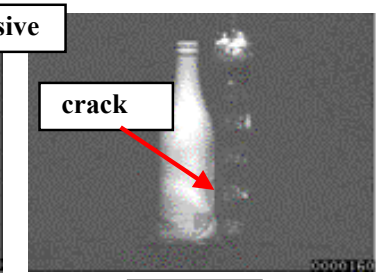

$160 \mu \mathrm{s}$

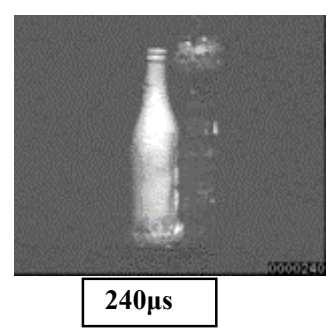

$240 \mu \mathrm{s}$

Figure 8: Shockwave propagation (sphere type). 

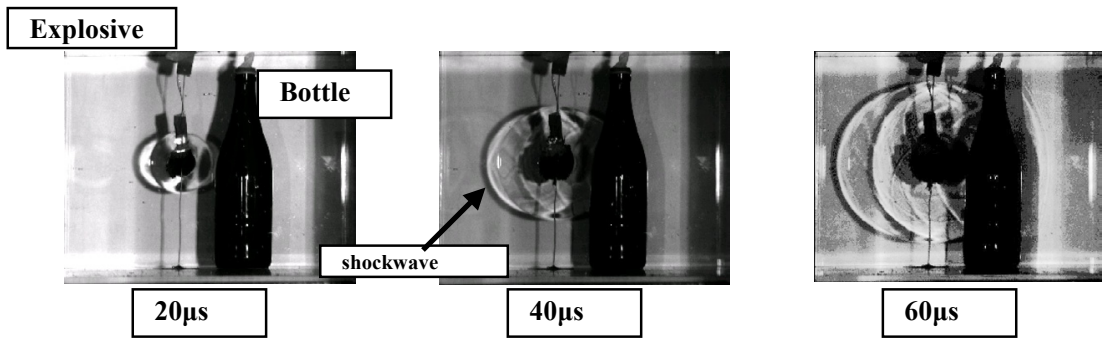

Figure 9: $\quad$ Fracture process by shockwave propagation (string type).

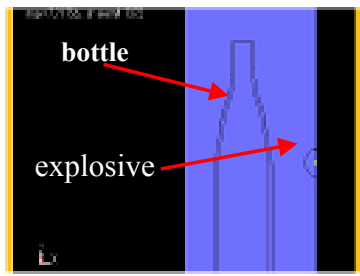

(a)

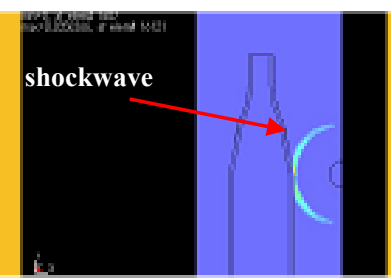

(b)

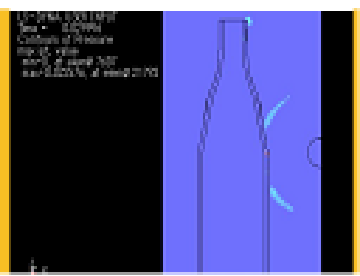

(c)

Figure 10: The propagation of underwater shockwaves by 2-D simulation in the case of sphere type.

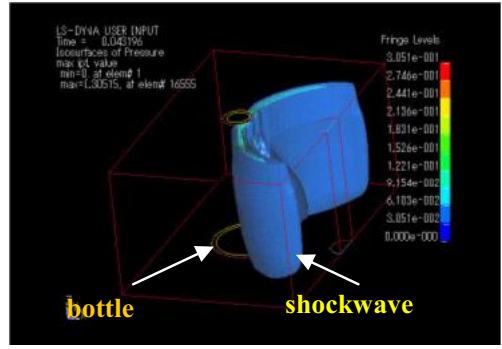

(a)

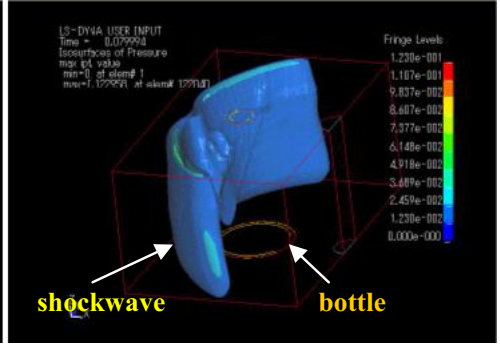

(b)

Figure 11: The behaviour of underwater shockwaves by LS-DYNA.

type. The fracture process of the glass bottle by underwater shockwaves is shown in Fig.9 in the case of the string type.

Figs.10-12 show the simulation results. In these analyses, LS-DYNA was used. Figure 10 shows the propagation of an underwater shockwave in the case of the sphere type (2-dimentinal analyses). The appearance of the shockwave propagation shows a good agreement with the experimental results.

Figure 11 shows the behaviour of an underwater shockwave by 3-dimensional analyses in the case of the string type. Figure 12 shows the appearance of the glass bottle crushing corresponding to the behaviour of the underwater shock wave shown in Fig.11. Good agreement between observed and calculated underwater shockwave behaviours was obtained. 


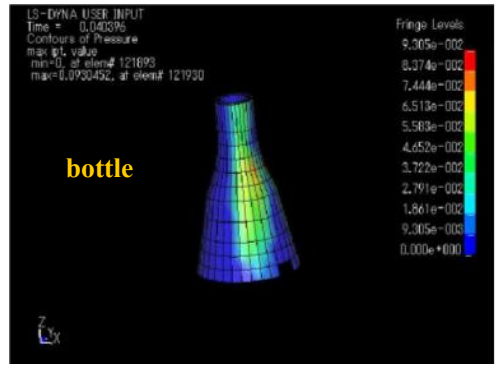

(a)

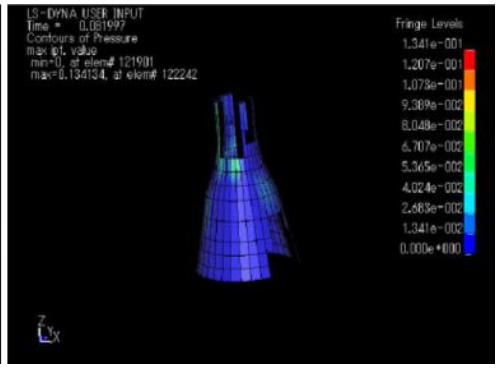

(b)

Figure 12: The fracture process of the glass bottle by LS-DYNA underwater shockwaves.

\section{Conclusions}

The relationship between the strength of underwater shockwaves and fracture cullet grain size was discussed. Moreover, the behaviours and the fracture process of the glass bottle underwater shockwaves were observed by the framing photograph method and the FEM simulation was executed in order to clarify the high-speed fracture mechanism.

The results obtained are summarized as follows:

1) The weight ratio of the small cullet grain sizes becomes higher as the distance of the bottle and the explosive shortens.

2) The behaviour of the underwater shockwave could be observed by optical methods.

3) The fracture process by underwater shockwaves and underwater shockwave behaviours were simulated by LS-DYNA and the analytical results were in good agreement with the experimental ones.

\section{References}

[1] S. Sakka, The dictionary of glass, Asakura Press Ltd., (1998).

[2] A.S. Kobayashi, Experimental Techniques in Fracture Mechanics, Society for Experimental Stress Analysis, (1973).

[3] V.P.W. Shim, S. Tanimura, C.T. Lim, Impact Response of Materials \& Structure, Oxford University Press (1999).

[4] H. Sakamoto, Computational Methods and Experimental Measurements VII, WIT Press (2005). P497.

[5] H. Sakamoto, Proc. of ESHP Workshop on Explosion, Shockwave and Hypervelocity Phenomenon, Kumamoto, Japan (2005), P90. 